\title{
ELONGACIÓN DE LA APÓFISIS ESTILOIDES Y EDENTULISMO EN MUJERES DE LA CIUDAD DE EL ALTO-BOLIVIA
}

\section{ELONGATION OF THE ESTILOIDES APOPHYSIS AND EDENTULISM IN WOMEN FROM EL ALTO-BOLIVIA}

\author{
Soto-Gonzales Fernando ${ }^{1 *}$, Cárdenas-López Juan ${ }^{2}$, Fajardo-Quezada Sandro ${ }^{2}$ \\ ${ }^{1}$ Especialista en Patología Buco Maxilofacial, Docente de Patología Estomatológica, Univerdidad particular de El Alto \\ UPEA, El Alto. Bolivia \\ 2 Odontólogo práctica privada. Cuenca. Ecuador \\ selaznogotos@hotmail.com
}

\section{Resumen}

\begin{abstract}
OBJETIVO: Determinar la frecuencia de la elongación de la apófisis estiloides en un grupo de mujeres de la ciudad de El Alto-Bolivia, y su posible relación con el edentulismo parcial y total. MATERIALES Y MÉTODOS: El estudio fue realizado en base al análisis de 100 radiografías panorámicas de pacientes de sexo femenino (de 35 a 73 años de edad), las mediciones de la longitud de las apófisis se realizaron mediante un software especializado. El punto de corte para el diagnóstico de elongación fue de 25mm. RESULTADOS: Se encontró una frecuencia total del $88 \%$ de elongación de la apófisis estiloides (sumando unilaterales y bilaterales). El grupo de edad con mayor frecuencia de elongación fue de las pacientes adultas jóvenes (18 a 44 años) con un presentaron elongación de las apófisis estiloides. La prueba de Chi-cuadrado permitió establecer una asociación estadísticamente significativa $(\mathrm{p}<0,05)$ entre el edentulismo y la elongación de la apófisis estiloides en el lado izquierdo del maxilar superior e inferior
\end{abstract}

Palabras clave: Síndrome de Eagle, arcada edéntula, frecuencia.

\begin{abstract}
AIM: To determine the frequency of elongation of the styloid process in a group of women from the city of El Alto-Bolivia, and its possible relationship with partial and total edentulism. MATERIALS AND METHODS: The study was conducted based on the analysis of 100 panoramic radiographs of female patients (from 37 to 74 years old), the measurements of the length of the apophysis were made using specialized software. The cut-off point for the diagnosis of elongation was $25 \mathrm{~mm}$. RESULTS: A total frequency of $88 \%$ elongation of the styloid process was found (adding unilateral and bilateral). The age group with the greatest frequency of elongation was young adult patients (18 to 44 years) with one elongation of the styloid processes. The Chi-square test allowed to establish a statistically significant association $(p<0.05)$ between edentulism and elongation of the styloid process on the left side of the upper and lower jaw
\end{abstract}

Key words: Eagle's syndrome, edentulous arcade, frequency.

\section{INTRODUCCIÓN}

El edentulismo parcial o total es definido por la Organización Mundial de la Salud (OMS) como la consecuencia de la destrucción o pérdida de piezas dentales. Esta condición provoca la alteración en las funciones básicas como el habla y la alimentación, ${ }^{1}$ afectando de manera significativa el desempeño diario de los edéntulos, alterando su función masticatoria, la oclusión dental y disminuyendo su calidad de vida. ${ }^{2}$
La elongación de la apófisis estiloides (es decir, con una longitud superior a 25-30mm) fue descrita en 1937 por W. Eagle $^{3}$, razón por la cual es conocida desde entonces con el nombre de Síndrome de Eagle. Se trata de una afección producto de la mineralización o calcificación de la apófisis (estilalgia), ${ }^{2}$ caracterizada por dolor sordo difuso, que se localiza a nivel craneocervical del ligamento estilo-hioideo. Pese a que se han propuesto diversas teorías para explicar su origen, en la actualidad se cree que la pérdida dental predispone a la elongación de la apófisis estiloides, lo que 
a su vez causaría su mineralización, debido a que con el paso del tiempo los tejidos pierden elasticidad. Esto tiene como consecuencia una inflamación localizada, que deriva en tendinitis ubicada en la unión del cuerno inferior del hioides y del ligamento, que luego causa la osificación del mismo. ${ }^{4}$ La ubicación particular de la apófisis estiloides en la parte inferior del peñasco del hueso temporal puede dar origen a procesos inflamatorios degenerativos que causan una tendinosis de la inserción estilohioidea provocando dolor. ${ }^{5}$ Esto se debe al contacto íntimo con algunas zonas anatómicas y nerviosas sensibles, como el nervio glosofaríngeo y la rama mandibular del trigémino. ${ }^{6,7}$

Existen casos en que los pacientes pueden o no presentar sintomatología, razón por la cual el tratamiento dependerá de la intensidad de los síntomas. En caso de dolor leve o moderado, se recomienda tratamiento con analgésicos habituales, dejando la opción quirúrgica para los casos de dolor intenso o recurrentes. $^{8-11}$

Estudios epidemiológicos reportan que la mineralización del ligamento estilohioideo se encuentra con mayor frecuencia en personas de sexo femenino, en un rango de edad que varía entre la tercera y la séptima década de vida. ${ }^{4-6,12,13}$ Sin embargo, en vista de que la mayoría de estudios se han realizado en población norteamericana y europea, surge la necesidad de conocer la frecuencia de este problema en países de otras regiones. Tal es el caso de Bolivia, donde no existe información acerca de la frecuencia de esta patología. Por todo lo anteriormente expuesto, el objetivo del presente estudio fue determinar la frecuencia de la elongación de la apófisis estiloides en mujeres de la ciudad de El AltoBolivia, mediante el uso de radiografías panorámicas. De igual manera se pretendía establecer una posible relación con el edentulismo parcial, así como establecer posibles diferencias según los rangos de edad de las pacientes. ${ }^{14,15}$

\section{MATERIALES Y MÉTODOS}

El estudio tuvo un diseño descriptivo transversal, observacional radiográfico. El tamaño de la muestra fue establecido por conveniencia y constó de 100 pacientes del sexo femenino, de 35 a 73 años de edad. La medición de la longitud de la apófisis estiloides, a partir de radiografías panorámicas, se realizó tomando en cuenta que las mismas tienen un rango de ampliación ligeramente mayor a la medida real; por esta razón, se aplicó un factor de corrección $(-2 \mathrm{~mm}),{ }^{16}$ considerándose que la apófisis estaba elongada cuando su longitud superaba los $25 \mathrm{~mm}$ de largo. ${ }^{17,18}$ La toma de los datos y su procesamiento se realizó en cuatro fases descritas a continuación:

- Fase 1: Se seleccionaron las pacientes de este estudio, familiares todas de los estudiantes del tercer año de la Universidad Pública de El Alto, durante los meses mayo-agosto del año 2014. • Fase 2: Se brindó a las pacientes la información sobre el trabajo de investigación, siguiendo los lineamientos éticos de la Asociación Médica Mundial, y se solicitó su consentimiento informado para realizar éste estudio. $\bullet$ Fase 3: Se registró la historia clínica de las pacientes, para lo cual se llevó a cabo el examen clínico intraoral para constatar la presencia de algunos signos clínicos (edentulismo total o parcial). - Fase 4: Finalmente se midió la longitud de la apófisis de cada paciente, previa toma de radiografías panorámicas, con la ayuda de un software especializado para la visualización y medición de imágenes (EasyDent V4 Viewer). Para realizar el análisis estadístico de los datos se agrupó a las pacientes en tres categorías de acuerdo al rango de edad: ${ }^{19}$ Adulta joven (de 18 a 44 años) Adulta media (de 45 a 64 años Adulta mayor (de 65 a más años)

Para analizar los datos se calculó la frecuencia de ocurrencia de apófisis elongadas en este grupo de pacientes; luego se realizó el mismo cálculo, subdividiendo a las pacientes en diferentes grupos de edades. Con la finalidad de evaluar la asociación entre variables, se empleó el estadístico Chicuadrado con un nivel de confiabilidad del $95 \%$.

\section{RESULTADOS}

En el presente estudio, la muestra total de 100 pacientes de sexo femenino se distribuyó en tres grupos etarios: adultas jóvenes $(n=24)$, adultas medias $(n=70)$ y adultas mayores $(n=6)$. La edad promedio de las pacientes fue de 50 años (D.E. 8,36); la mínima de 35 años y la máxima de 73 años. Se encontró una frecuencia total del $88 \%$ de elongación de la apófisis estiloides (sumando unilaterales y bilaterales). El $74 \%$ de las pacientes adultas medias presentaron apófisis estiloides elongadas (mayor a $25 \mathrm{~mm}$ ) en el lado derecho, y $77 \%$ en el lado izquierdo (Tabla 1). Comparando todos los grupos de edad la mayor frecuencia de apófisis elongada se halló en pacientes adultas jóvenes.

Por otro lado del total de personas edéntulas parciales del maxilar superior, el $78 \%$ presentaron apófisis estiloides elongadas en el lado derecho, mientras que $83 \%$ lo hicieron en el lado izquierdo (Tabla 2). En cuanto a las pacientes edéntulas parciales del maxilar inferior, el $78 \%$ presentó elongación en el lado derecho y $82 \%$ en el lado izquierdo (Tabla 2).

Respecto a las pacientes edéntulas totales de maxilar superior el $61 \%$ presentaron apófisis elongada en el lado derecho mientras que el $70 \%$ lo hicieron en el lado izquierdo (Tabla 3). En pacientes con edentulismo total inferior, el $64 \%$ presentaron elongación de la apófisis del lado derecho y $55 \%$ en el lado izquierdo (Tabla 3). La prueba de Chicuadrado permitió establecer una asociación estadísticamente significativa $(\mathrm{p}<0,05)$ entre el edentulismo y la elongación de la apófisis estiloides en el lado izquierdo del maxilar, tanto superior como inferior (Tablas 2 y 3 ) 


\begin{tabular}{ccccccccccc}
\multicolumn{1}{c}{ ELONGACIÓN DE } & \multicolumn{7}{c}{ ELONGACIÓN DE } \\
& \multicolumn{1}{c}{ APÓFISIS DERECHA } & \multicolumn{2}{c}{ APÓFISIS IZQUIERDA } \\
& \multicolumn{2}{c}{ Sano } & Elongado & \multicolumn{2}{c}{ Sano } & \multicolumn{2}{c}{ Elongado } & Total \\
& $\mathrm{n}$ & $\%$ & $\mathrm{n}$ & $\%$ & $\mathrm{n}$ & $\%$ & $\mathrm{n}$ & $\%$ & $\mathrm{n}$ & $\%$ \\
Adulto jóven & 4 & $17 \%$ & 20 & $83 \%$ & 3 & 12 & 21 & $88 \%$ & 24 & $24 \%$ \\
Adulto medio & 18 & $26 \%$ & 52 & $74 \%$ & 17 & 23 & 54 & $77 \%$ & 70 & $70 \%$ \\
Adulto mayor & 3 & $50 \%$ & 3 & $50 \%$ & 2 & 33 & 4 & $67 \%$ & 6 & $6 \%$ \\
Total & 25 & $25 \%$ & 75 & $100 \%$ & 21 & 21 & 79 & $79 \%$ & 100 & $100 \%$ \\
\hline
\end{tabular}

Tabla 1. Frecuencia de la elongación de la apófisis estiloides según rango de edad

\begin{tabular}{|c|c|c|c|c|c|c|c|c|c|c|}
\hline & \multicolumn{4}{|c|}{$\begin{array}{c}\text { ELONGACIÓN DE } \\
\text { APÓFISIS DERECHA }\end{array}$} & \multicolumn{3}{|c|}{$\begin{array}{c}\text { ELONGACIÓN DE } \\
\text { APÓFISIS IZQUIERDA }\end{array}$} & & & \\
\hline & \multicolumn{2}{|c|}{ Sano } & \multicolumn{2}{|c|}{ Elongado } & \multicolumn{2}{|c|}{ Sano } & \multicolumn{2}{|c|}{ Elongado } & \multicolumn{2}{|c|}{ Total } \\
\hline & $\mathrm{n}$ & $\%$ & $\mathrm{n}$ & $\%$ & $\mathrm{n}$ & $\%$ & $\mathrm{n}$ & $\%$ & $\mathrm{n}$ & $\%$ \\
\hline Dentado & 1 & $13 \%$ & 7 & $87 \%$ & 2 & 25 & 6 & $75 \%$ & 8 & $8 \%$ \\
\hline Edéntulo parcial & 15 & $22 \%$ & 54 & $78 \%$ & 12 & 17 & 57 & $83 \%$ & 69 & $69 \%$ \\
\hline Edéntulo total & 9 & $39 \%$ & 14 & $61 \%$ & 7 & 30 & 16 & $70 \%$ & 23 & $23 \%$ \\
\hline Total & 25 & $25 \%$ & 75 & $75 \%$ & 21 & 21 & 79 & $79 \%$ & 100 & $100 \%$ \\
\hline
\end{tabular}

Chi-cuadrado $\mathrm{p}=<0,17 \quad$ Chi-cuadrado $\mathrm{p}=<0,03$

Tabla 2. Frecuencia de la elongación de la apófisis estiloides en edentulismo superior

\begin{tabular}{|c|c|c|c|c|c|c|c|c|c|c|}
\hline & \multicolumn{4}{|c|}{$\begin{array}{c}\text { ELONGACIÓN DE } \\
\text { APÓFISIS DERECHA }\end{array}$} & \multicolumn{3}{|c|}{$\begin{array}{c}\text { ELONGACIÓN DE } \\
\text { APÓFISIS IZQUIERDA }\end{array}$} & & & \\
\hline & \multicolumn{2}{|c|}{ Sano } & \multicolumn{2}{|c|}{ Elongado } & \multicolumn{2}{|c|}{ Sano } & \multicolumn{2}{|c|}{ Elongado } & \multicolumn{2}{|c|}{ Total } \\
\hline & $\mathrm{n}$ & $\%$ & $\mathrm{n}$ & $\%$ & $\mathrm{n}$ & $\%$ & $\mathrm{n}$ & $\%$ & $\mathrm{n}$ & $\%$ \\
\hline Dentado & 0 & $0 \%$ & 1 & $100 \%$ & 0 & 0 & 1 & $100 \%$ & 1 & $1 \%$ \\
\hline Edéntulo parcial & 21 & $22 \%$ & 67 & $78 \%$ & 16 & 18 & 72 & $82 \%$ & 88 & $88 \%$ \\
\hline Edéntulo total & 4 & $36 \%$ & 7 & $64 \%$ & 5 & 45 & 6 & $55 \%$ & 11 & $11 \%$ \\
\hline Total & 25 & $25 \%$ & 75 & $75 \%$ & 21 & 21 & 79 & $79 \%$ & 100 & $100 \%$ \\
\hline
\end{tabular}

Tabla 3. Frecuencia de la elongación de la apófisis estiloides en edentulismo inferior

\section{DISCUSIÓN}

El síndrome de Eagle es una patología poco conocida en nuestro medio; por ello consideramos relevante conocer la frecuencia de elongación de la apófisis estiloides, El presente estudio descriptivo observacional, clínico y radiológico determinó una frecuencia total de $77 \%$ de elongación de la apófisis estiloides. 
Es importante destacar que estas elongaciones pueden identificarse como un hallazgo casual en las radiografías panorámicas, simulando otras patologías distintas. Es necesario que el clínico sepa identificarlas y de esta manera diagnosticar la enfermedad y no confundirla con otras patologías. ${ }^{20,21}$ Los datos del presente estudio revelaron que es en lado derecho del cráneo donde se presenta la elongación de la apófisis estiloides con mayor frecuencia. Esto confirma resultados de estudios previos como los de Langlais $^{2}$ y Machado. $^{22}$ Por otro lado, los resultados obtenidos en nuestro estudio evidencian una mayor frecuencia de apófisis elongada en pacientes adultas jóvenes. Esto coincide con el estudio de Gomes, ${ }^{15}$ quien reportó una mayor frecuencia de mineralización de la apófisis estiloides en adultos jóvenes. Por el contrario, difiere de lo que se ha reportado en la mayoría de los estudios. Por ejemplo, Rizzatti-Barbosa y cols., ${ }^{23}$ determinaron una mayor frecuencia de esta patología en adultos medios, mientras que Matilla y cols. ${ }^{24}$ reportan una mayor prevalencia en la población adulta mayor que presenta, además, edentulismo parcial. Una posible limitación del estudio, es la conformación de la muestra con pacientes exclusivamente de sexo femenino, lo cual no permite la comparación de las frecuencias de acuerdo al sexo. En este estudio se conformó la muestra de esta forma, debido a que al inicio del estudio no había la certeza de encontrar ni un solo caso de esta condición, y debido a que la literatura indica que en otras latitudes es mayor la frecuencia en mujeres se decidió iniciar este estudio descriptivo exploratorio para tener un primer acercamiento al fenómeno. Otra limitación del estudio es que se empleó la radiografía panorámica para la medición, esto puede tener un porcentaje de error (que se trató de menguar con el factor de corrección). Este trabajo destaca por su originalidad local, siendo el primer acercamiento al estudio de este fenómeno en población de Bolivia, lo cual nos permitirá iniciar una línea de investigación y vigilancia epidemiológica respecto a esta patología y es de suma importancia debido a que los valores hallados de frecuencia, son de utilidad para poder iniciar el cálculo muestral en futuros estudios poblacionales, tal como lo propone Villavicencio E y Corrales-Reyes. ${ }^{25,26}$ Todo lo anterior nos permite proponer que la elongación de la apófisis estiloides se debe a la pérdida prematura de piezas dentales en edades tempranas, lo cual a su vez está estrechamente relacionado con los altos índices de caries, que son característicos en esta región. ${ }^{27}$

CONFLICTO DE INTERESES: Los autores no tienen ningún conflicto de interés.

AGRADECIMIENTOS: Los Autores agradecen a: - Dr. Luis Andrés Yarzábal (Departamento de Investigación, Carrera de Odontología, Universidad Católica de Cuenca) por su apoyo en la edición y la revisión crítica del manuscrito - Dr. Ebingen Villavicencio Caparó (Departamento de Inves- tigación, Carrera de Odontología, Universidad Católica de Cuenca) por la ayuda en la revisión del presente artículo.

- Ing. Rodolfo Barbeito por su ayuda en la diagramación del presente artículo.

\section{Referencias}

1 Organización Mundial de la Salud. La OMS publica un nuevo informe sobre el problema mundial de las enfermedades bucodentales. [Online].; 2004 [cited 2018 Enero 21. Available from: https://www.who.int/mediacentre/news/releases/2004/pr15/es/.

2 Langlais P, Miles D, Van-Dis M, Elongated and mineralized stylohyoid ligament complex: A proposed classification and report of a case of Eagle's Syndrome. Oral Surgery, Oral Medicine, Oral Pathology. 1986; 61, 527-532.

3 Eagle W. Elongated styloid process. Further Observation and a New Syndrome. Arch. Otolaryngol. 1948; 47: 630-40.

4 Feldman V, Eagle's syndrome: a case of symptomatic calcification of the stylohyoid ligaments. The Journal of the Canadian Chiropractic Association. 2003. 47 (1): 21-27.

5 Rouvière H, Delmas V. Anatomía Humana Descriptiva, Topográfica, Funcional, $11^{\circ}$ Edición Tomo I, Editorial Aleu S.A. 2005: 69-71

6 Testut L. Tratado de Anatomía Humana, $8^{\text {a }}$ Edición. Tomo I. Editorial Salvat, 1969. 153-168.

7 O Rahilly R. Anatomía De Gardner, 5ta. edición cap.52, (Ed.) Editorial interamericana Mcgaw-Hill 1989: 647-670.

8 Carlson M; Embriología humana y biología del desarrollo, $2^{a}$ Edición, (Ed) Harcourt, 2000: 292-298

9 Langman J; Embriología Médica; $10^{\mathrm{a}}$ ed. (Ed.). Médica Panamericana.1981: 335-369.

10 Raffo M. Síndrome de Eagle: reporte de un caso. Odontoestomatol. 2012; 14(20): 26-31. Disponible en: http:// www.scielo.edu.uy/scielo.php?script=sci_arttext\&pid=S168893392012000200004

11 Correll R, Jensen J, Taylor J, Rhyme RR. Mineralization of the stylohyoid ligament complex. Oral Surg Oral Med Oral Pathol. 1979;48(4):286-291.

12 Fuentes R, Oporto G, Garay I, Bustos L, Silva H, Flores H. Proceso Estiloides en una Muestra de Radiografías Panorámicas de la Ciudad de Temuco-Chile. Int. J. Morphol. 2007 Dec; 25(4): p. 729-733.

13 Sánchez E, Nary H, Casati L. Eagle's syndrome: radiographic study of the incidence of elongated styloid process. Salusvita, Bauru. 2003; 22(1): p. 25- 33.

14 Garay I, Olate S. Osificación del Ligamento Estilohioideo en 3.028 Radiografías Panorámicas Digitales. Int. J. Morphol. 2013; 31(1): p. 31-37.

15 Gomes W, Nascimento G, Vessoni L, Chicarelli M, Amosoro P, Souza L. Prevalencia de alteraciones en el complejo estilohiodeo en radiografías panorámicas digitales, $\mathrm{Rev} \mathrm{Cu}$ bana Estomatol. 2015;52(2): 1-7

16 Goaz P, Stuart C. Radiología oral: principios e interpretación. 2018; 3(1): 629-631. 
17 Tísner J, Tísner B, Abad J, Abad A, Calcificación del ligamento estilohioideo: estilalgia de Aubin y síndrome de Eagle. Aportación de 5 casos clínicos. Sociedad Aragonesa de Otorrinolaringología. 2003; 6(2): 5-12

18 Camarda A, Deschamps C, Forest D. Stylohyoid chain ossification: A discussion of etiology. Oral Surg Oral Med Oral Pathol. 1989, 67(5): 508-514.

19 Cardona D, Agudelo HB, Tendencias de mortalidad en población adulta, Medellín, 1994-2003. Biomédica [en linea] 2007, 27 (septiembre) : [Fecha de consulta: 21 de enero de 2018] Disponible en: http://www.redalyc.org/articulo.oa?id=84327305

20 Padrós E, Implicaciones miofuncionales en el síndrome de Eagle. Bases diagnósticas, terapéuticas y posturales del funcionalismo cráneo mandibular. 2002: 276- 278.

21 Morales E, Cantín L, Proceso estiloides elongado: Criterios diagnósticos y diagnóstico diferencial. A propósito de un caso. Int. J. Odontostomat. 2010; 4(3): 223-228.

22 Machado K. Styloid Process Elongation and Calcification. Braz Dent J. 2012; 23(4): p. 443-450.

23 Rizzatti-Barbosa C, Ribeiro M, Silva-Concilio L, Di Hipolito $\mathrm{O}$, Ambrosano $\mathrm{G}$. Is an elongated stylohyoid process prevalent in the elderly? A radiographic study in a Brazilian population. Gerodontology. 2005; 22(2): 112-115.

24 Matilla M, García E, Cañón D, Mantilla A, Rojas J, Londoño D. Sociedad Española de Radiología Médica. Seram. 2014; 56. 1-228.

25 Villavicencio E. La importancia de los estudios descriptivos. Rev Evid Odontol Clinic [Internet]. 2016 [citado 14 de abril de 2018];2(1):6-7. Disponible en: https:// uancv.edu.pe/revistas/index.php/EOC/article/viewFile/77/59

26 Corrales-Reyes IE, Fornaris-Cedeño Y, Dorta-Contreras AJ, Mejia CR. Baja publicación de los trabajos presentados en el IV Encuentro Iberolatinoamericano de Estudiantes de Odontología. Rev. cuba. inf. cienc. Salud [Internet]. 2018;Sep [citado 2019 Ene 21] ; 29(3): 1-14. Disponible en: http://scielo.sld.cu/scielo.php?script=sci_arttext\&pid=S230721132018000300004\&lng=es.

27 Ministerio de Salud Pública de Bolivia, Programa Nacional de Salud Oral (PNSO) 2016.Disponible en: https://www.minsalud.gob.bo/2144-ministerio-de-saludcoadyuvo-en-reduccion-del-indice-de-poblacion-conproblema-de-caries-en-los-dientes

Recibido: 28 de Agosto de 2018

Aceptado: 29 de Septiembre 2018 
